\title{
All Optical Multicast Routing in Sparse-Splitting Optical Networks
}

\author{
Cheng-Yu Hsieh ${ }^{1}$ and Wanjiun Liao \\ Department of Electrical Engineering \\ National Taiwan University \\ Taipei, Taiwan \\ Email:wjliao@cc.ee.ntu.edu.tw
}

\begin{abstract}
This paper studies all-optical multicast routing in wavelength-routed optical networks with sparse light splitting. In a sparse splitting network, only a small percentage of nodes are capable of light splitting, i.e., multicast capable. The typical solutions of existing multicast routing algorithms for sparse splitting networks combine an existing Steiner Tree heuristic with some rerouting procedures to refine the trees. The resulting tree cost in terms of the total number of wavelengths used on all tree links is then very expensive. In this paper, we propose a new mechanism that constructs all-optical multicast trees for sparse splitting networks without an additional rerouting procedure in the tree construction. Two efficient approaches are suggested and evaluated by simulations. The results show that our mechanism builds light-trees with the least wavelength channel cost and with the smallest number of wavelengths used per link.
\end{abstract}

\section{Introduction}

Multicast is an efficient way for one-to-many or many-to-many communications. A multicast session typically involves a source and a set of receivers (destinations). To avoid sending a separate copy to each of the receivers, a multicast session is typically implemented with a multicasting tree, with the root at the source and the branches spanning all the receivers.

The multicast problem in IP networks has been studied for years and many efficient multicast routing protocols have been developed. To support multicast at the Wavelength-Division Multiplexing (WDM) layer, [1] introduced the concept of the light-tree, which is a point-to-multipoint extension of a lightpath (i.e., an all-optical WDM channel). The key advantage of light-trees is that only one transmitter is needed for transmission and intermediate tree links can be shared by

\footnotetext{
${ }^{1}$ This author is now with Quanta Computer Inc. in Taiwan.
}

multiple destinations. To support all-optical multicasting efficiently, nodes in a WDM network need to have the light (optical) splitting capability. A node with splitting capability can forward an incoming message to multiple output channels, and therefore is multicast-capable (MC). An MC node, however, is expensive to implement. The concept of sparse-splitting was first introduced in [2]. With sparse splitting, only a small percentage of nodes in the network are Multicast Capable, and the rest are Multicast Incapable (MI). An MI node can forward an input signal only to one of the output ports; thus it cannot serve as a branching node of a light-tree.

Route optimization in sparse-splitting networks is discussed in [2-5]. In [2], four heuristics are proposed to generate multiple light-trees (i.e., light forest) for a given multicast session, thus using multiple transmitters and multiple wavelengths per session. In [3], a solution which generates a light-tree using single transmitter and single wavelength per session is presented. In [4], an all-optical multicast routing heuristic called AOMH is proposed to generate a light-tree using single transmitter and multiple wavelengths per session, with the assumption that MI nodes are equipped with Tap-or-Continue (ToC) cross-connects. A ToC cross-connect cannot tap optical power from a wavelength channel for the local router when data are forwarded to an output channel. The cross-connect that allows optical power to be tapped while forwarding data to an output is called Tap-and-Continue ( $\mathrm{TaC})$. In [5], a virtual source-based multicast routing scheme is suggested. The mechanism establishes mutual connectivity among all MC nodes in advance.

Existing multicast solutions for sparse splitting WDM networks can be classified into two types: source-based trees [2-3] and MC-based trees [4-5]. Source-based multicast schemes build light-trees in a way similar to traditional IP multicast trees, with modifications to the routing algorithm for adapting to the sparse-splitting constraint. In MC-based schemes, a light-tree connecting 
the MC nodes of a multicast group is first constructed, called $M C$-tree. Each MI node of the group then separately joins the nearest $\mathrm{MC}$ node which is already on-tree. Compared to source-based tree approaches, MC-based schemes have better resource utilization, better support for dynamic membership, shorter tree setup latency, and are more scalable to large networks [5]

The efficiency of an all-optical multicast routing mechanism can be evaluated based on the tree cost of the corresponding multicast session. Such a cost includes the number of wavelength channels required and the maximum number of wavelengths used per link. For a multicast session, the number of wavelength channels required is defined as the total number of wavelengths used over all links on the tree (i.e., the total number of multicast tree branches). The cost of constructing a multicast tree can be greatly reduced when nodes are capable of light splitting. Further cost reduction can be achieved if $\mathrm{MI}$ nodes support $\mathrm{TaC}$ instead of $\mathrm{ToC}$. However, $\mathrm{TaC}$ operations may not be supported in most wavelength switch architectures unless an add-drop multiplexer is used to receive packets through $\mathrm{O} / \mathrm{E}$ and forward copies through E/O [4]. Thus, for all-optical multicasting, it is more reasonable to assume that MI nodes support $\mathrm{ToC}$, rather than $\mathrm{TaC}$.

It is important to minimize the number of wavelength channels for a multicast session because the number of wavelengths supported in a fiber link is limited. In this paper, we will focus on developing an all-optical multicast routing algorithm that minimizes the number of wavelength channels required by a given multicast session. We attempt to build a light-tree with minimum cost for a given multicast session. The cost of a light-tree here is defined as the total number of wavelength channels used by a light-tree. In MC-based multicast routing schemes, the cost of a resulting light-tree is dominated by the cost of the corresponding $\mathrm{MC}$ tree. This is because such a cost equals the cost of the MC-tree plus that of each light-path from an MI node to the MC-tree. In addition, the cost of MI nodes connecting to the MC-tree also depends on where the nearest $\mathrm{MC}$ nodes of the MC-Tree are located. Thus, it is crucial to find the minimum-cost MC-tree in order to minimize the total cost of the resulting light-tree.

Existing multicast routing algorithms for sparse-splitting networks usually include two phases of tree construction. First, an existing Steiner Tree heuristic is used to obtain an initial tree without taking the sparse-splitting constraint into account, and then some rerouting procedure is applied to refine the tree (see [2-4] for example). Consequently, the resulting tree is expensive because it is equal to the sum of the cost of the original tree constructed by a Steiner Tree heuristic and an additional cost due to rerouting, denoted as rerouting cost.

In this paper, we propose a new all-optical multicast routing algorithm to construct light-trees without any additional rerouting procedure. We suggest two efficient approaches to growing a light-tree for a given multicast session in which the tree cost is minimized. We then perform simulation to compare the performance of our mechanism with existing work. The results show that our mechanism builds light-trees with the least wavelength channel cost and with the smallest number of wavelengths used per link for a session.

The rest of the paper is organized as follows. Section 2 describes the operation of minimum-cost MC-tree construction, and presents two efficient mechanisms for light-tree construction. Section 3 shows the simulation results. Section 4 concludes the paper.

\section{All-Optical Light-Tree Construction}

In this section, we describe the proposed mechanism for all-optical multicast routing in wavelength-routed optical networks with sparse splitting. We adopt the MC-based scheme for tree construction, but eliminate the extra rerouting procedure. In what follows, we first describe how the proposed mechanism constructs an MC-tree with minimum cost for a given multicast session, and then present two different policies of selecting MC nodes for MI nodes to join the tree.

\subsection{Problem Statements}

\section{Assumptions}

The optical network we consider is assumed to coexist with two types of cross-connects: MI and MC nodes. MI nodes perform ToC operations during data forwarding, while $\mathrm{MC}$ nodes support light splitting, forwarding data to multiple wavelength channels. MC nodes are assumed equipped with wavelength converters. Multicast receivers may be connected to MC or MI nodes.

\section{Problem Statements}

The sparse splitting optical network can be modeled as a directed connected graph $\mathrm{G}(\mathrm{V}, \mathrm{E})$, where $\mathrm{V}$ and $\mathrm{E}$ are the sets of nodes and directed links of the network, respectively. Let $\Lambda(e)=\left\{\lambda_{1}, \lambda_{2}, \cdots, \lambda_{k}\right\}$ denote the set of wavelengths supported on link $e$ in the network. A multicast request is denoted $\mathrm{r}(\mathrm{s} ; \mathrm{D})$, where $s$ is the source 
node and $D$ is the set of destinations.

Given network $\mathrm{G}, \Lambda(e)$ for every $\mathrm{e} \in \mathrm{E}$, and a multicast session request $r(s ; D)$, finding a multicast tree such that the tree cost is minimized can be proved to be NP-hard. This is because minimizing tree cost can be regarded as minimizing the total number of wavelength channels used on the tree. In other words, this problem is equivalent to developing a multicast routing algorithm which minimizes total wavelength channel cost under the constraint of sparse light-splitting capability. Finding a multicast routing algorithm such that the total wavelength channel cost is minimized is NP-hard because it can be reduced to the standard Steiner Tree problem when the sparse-splitting constraint is relaxed.

This paper focuses on constructing an all-optical multicast tree for a given multicast session such that the total number of wavelength channels required is minimized. Our tree is comprised of a single light-tree, thus using single transmitter and multiple wavelengths per session, in sparse-splitting WDM networks. We adopt MC-based multicast approach, in which a light-tree is comprised of an $\mathrm{MC}$ tree and a set of lightpaths connecting MI nodes to the MC tree. In the following, we first describe how a minimum-cost $\mathrm{MC}$ tree is grown, and then extend the procedure to a complete light-tree with both $\mathrm{MC}$ and MI nodes, using two different approaches.

\subsection{Minimum-Cost MC-Tree}

For MC-based multicast schemes, the resulting light-tree, say $\mathrm{T}$, consists of an MC-Tree $\mathrm{T}_{\mathrm{MC}}$ and a set of lightpaths $\mathrm{L}_{\mathrm{T}}$ to connect $\mathrm{MI}$ nodes with the remaining destinations to the MC Tree. Thus, the wavelength channel cost of tree $\mathrm{T}$, denoted as $\mathrm{WC}(\mathrm{T})$, is equal to the wavelength channel cost of the MC tree plus that of the lightpaths, i.e., $W C(T)=W C\left(T_{M C}\right)+\sum_{L_{T}^{i} \in L_{T}} W C\left(L_{T}^{i}\right)$. Our

design goal here is to develop an efficient algorithm that minimizes $W C\left(T_{M C}\right)$. This problem is, however, NP-hard because it can be reduced to the standard Steiner tree problem when the sparse-splitting constraint is relaxed.

The standard approach of existing work (e.g., [2-5]) to constructing a light-tree is to combine an existing IP multicast routing algorithm with some rerouting procedures. Here we aim at eliminating the extra rerouting for light-tree construction. In our solution, an auxiliary $M C$ network $\mathrm{M}_{\mathrm{G}}$ is first built from the original network $\mathrm{G}$, and then an existing Steiner tree heuristic is applied to $\mathrm{M}_{\mathrm{G}}$ to obtain a minimum-cost $\mathrm{MC}$ tree. The $M C$ network $\mathrm{M}_{\mathrm{G}}$ in $\mathrm{G}(\mathrm{E}, \mathrm{V})$ is defined as a network, in which

(1) All MC nodes in G(E,V) are included as its vertex set, say W.

(2) Two $\mathrm{MC}$ nodes in $\mathrm{M}_{\mathrm{G}}$ are connected if there is a path between these two MC nodes in $\mathrm{G}(\mathrm{E}, \mathrm{V})$.

(3) Link cost from node $i$ to node $j$ in $\mathrm{M}_{\mathrm{G}}$ is equal to the cost of the minimum-weight path from node $i$ to node $j$ in $\mathrm{G}$, for all $i, j \in \mathrm{W}$.

In [6], we have proved that finding an $\mathrm{MC}$ tree with minimum wavelength channel cost is equivalent to finding a Steiner tree in the corresponding MC network. Thus, once an auxiliary $\mathrm{MC}$ network is constructed, we can apply any existing Steiner Tree heuristic for a multicast session in the MC network to obtain the minimum-cost MC Tree. The MC-Tree is then transformed back to the original network to yield the resulting light-tree. We call such an approach "Auxiliary Network Transformation (ANT)," which is summarized as follows (as shown in Fig. 1).

1. The MC network $\boldsymbol{M}_{\boldsymbol{G}}$ of the original network $\boldsymbol{G}$ is determined.

2. A Steiner-tree heuristic is applied to $\boldsymbol{M}_{\boldsymbol{G}}$ to generate a minimum-cost tree $\mathbf{T}_{\mathbf{R}}$ for multicast request $\mathrm{R}$.

3. The resulting light-tree $\mathrm{T}$ is obtained by substituting each link, say $(i, j)$, in $\mathbf{T}_{\mathbf{R}}$ with the corresponding minimum-cost path from node $i$ to node $j$ in $G$.

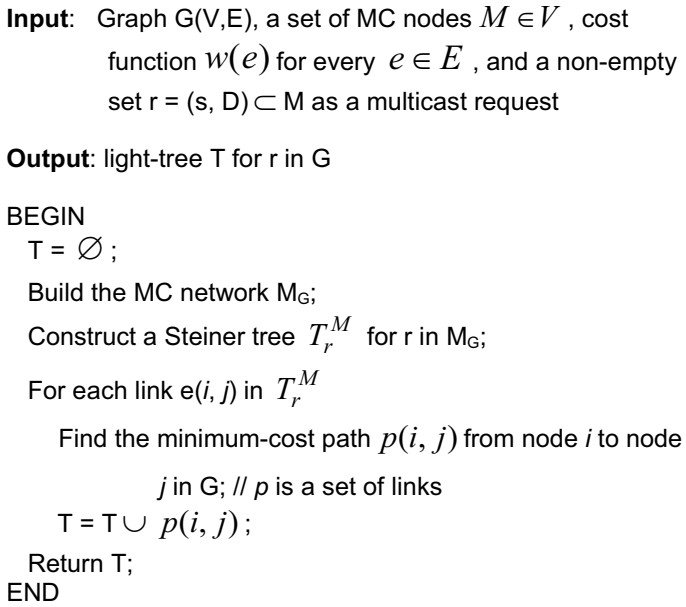

Figure 1. ANT

Fig. 2 gives an example to demonstrate how ANT works. The circles denote MI nodes while the squares, MC nodes. Node $\mathrm{s}$ is the source and the other shaded nodes are destinations. Fig. 2 (b) depicts the resulting MC-tree with a cost of 11 by AOMH. The corresponding MC network is shown in Fig. 2 (c), and the resulting MC-tree with a cost of 8 by ANT is shown in Fig. 2(d). 


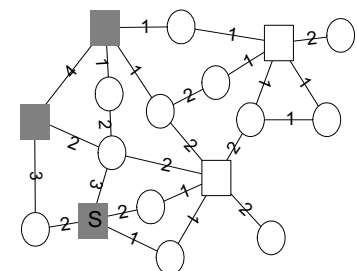

(a) Network topology

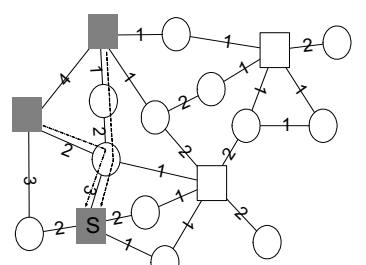

(b) Resulting light-tree generated by $\mathrm{AOMH}$

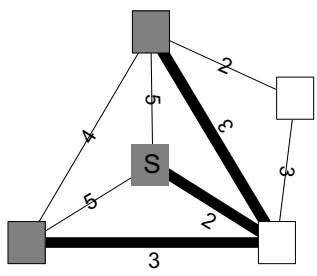

(c) The corresponding MC network and resulting Steiner tree

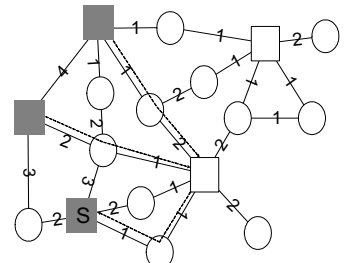

(d) Resulting light-tree generated by ANT

Figure 2. An example of ANT operation

\subsection{The Selection Policy of MC Nodes}

In the previous section, we have described how ANT determines a minimum-cost $\mathrm{MC}$ tree for a multicast request, under which the $\mathrm{MC}$ nodes to be included are given. This section returns to the issue of selecting MC nodes to be included. Recall that in Section 1, we stated that the number of MC nodes included on an MC-tree not only affects the cost of MC-tree itself, but also the cost of MI nodes connecting to the MC-tree. The more $\mathrm{MC}$ nodes are involved in an MC-tree, the more resource is used to construct the MC-tree. On the other hand, the lightpath cost from an MI node to the MC-tree may be reduced if the MC-tree spans all over the network. Consequently, the selection policy of MC nodes by MI nodes to join the MC-tree is key to determining the cost of the resulting light-tree. It is hard to optimally select a set of MC nodes (to grow an MC-Tree) which minimizes the cost of the resulting light-tree for a given multicast session. Since this problem is equivalent to finding an MC-tree with minimum wavelength channel cost when all group members are connected to MC nodes, again, it is an NP-hard problem. Thus, it is required to find an efficient approach that chooses a suitable subset of MC nodes in the network as on-tree MC nodes.

In the following, we propose two efficient approaches to selecting MC nodes as on-tree MC nodes, based on two different design philosophies.

(1) The On-Tree MC node First (OTMCF) mechanism attempts to minimize the cost of an MC-tree. Thus an MC-tree is constructed first by connecting all the MC nodes with group members, and then is expanded as follows. MI nodes with the remaining members join the tree at the nearest on-tree MC nodes.

(2) The Nearest MC node First (NMCF) approach is designed to minimize the cost of MI nodes joining the MC-tree. The set of MC nodes used to construct an MC-tree includes all MC nodes directly connecting destinations of the group and the MC nodes nearest to each MI nodes with the remaining destinations. NMCF expands on-tree MC nodes in a way that each $\mathrm{MC}$ node nearest to each MI node in the group should also be on-tree.

Fig. 3 summarizes the operations of light-tree construction by NMCF and OTMCF, in conjunction with ANT described in Section 2.2.

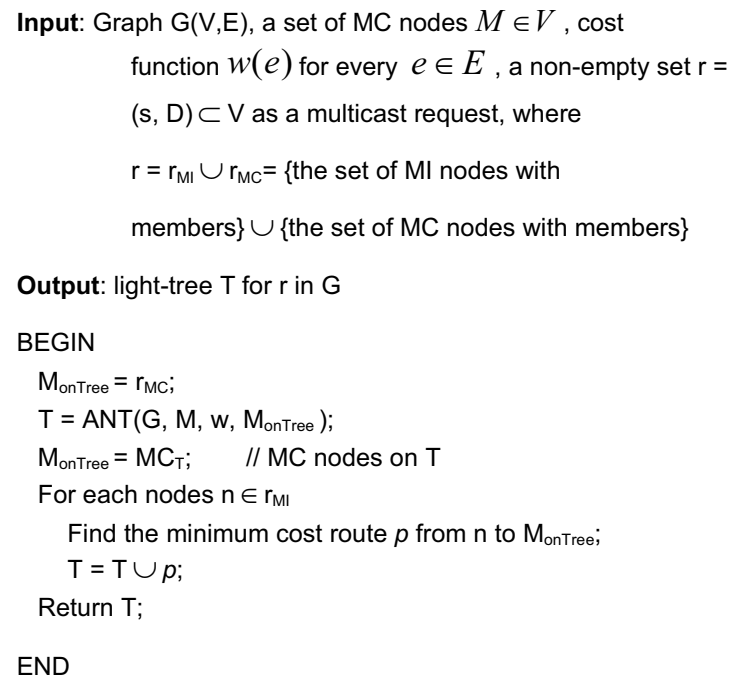

(a) NMCF 


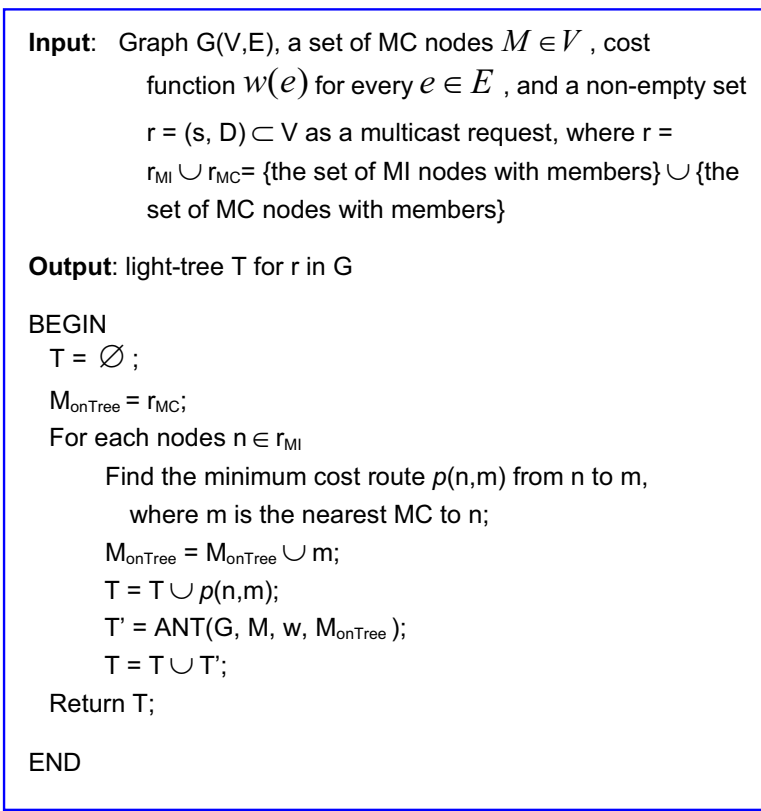

(b) OTMCF

Figure 3. Two MC-node selection policies

\section{Performance Evaluation}

In this section, we present the simulation results to compare the performance of OTMCF, NMCF with AOMH and MemberOnly. We use KMB as the Steiner-tree heuristics for ANT and AOMH in the simulation. A flat network with 50 nodes is generated with GT-ITM. Each MC node in the network is assumed to be equipped with both splitting and wavelength conversion capabilities. The cost of each wavelength channel on each link is assigned one.

\subsection{Wavelength Channel Cost}

This experiment is to compare the performance of light-trees constructed by MC-tree based mechanisms i.e., $\mathrm{OTMCF}, \mathrm{NMCF}$ and $\mathrm{AOMH}$, varying the fraction of $\mathrm{MC}$ nodes from 0.1 to 0.8 . Fig. 4 shows wavelength channel cost of light-trees (i.e., the average number of wavelength channels needed for establishing a multicast session) for the three approaches. We show three sets of results, with group sizes 10,20 , and 30 , respectively. In any case, both OTMCF and NMCF have better performance than AOMH. Besides, NMCF outperforms OTMCF.

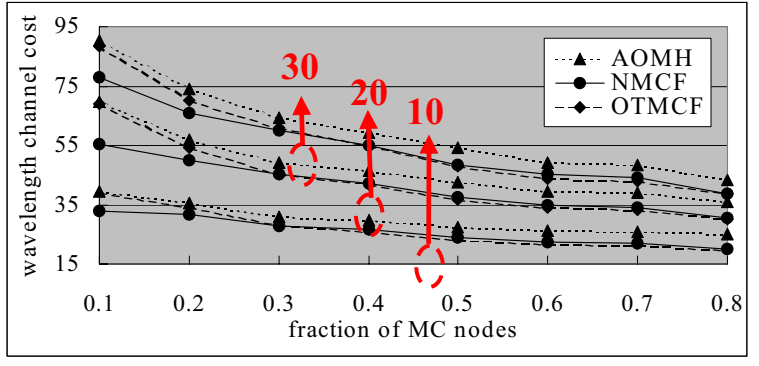

Figure 4. Wavelength channel cost of a light-tree

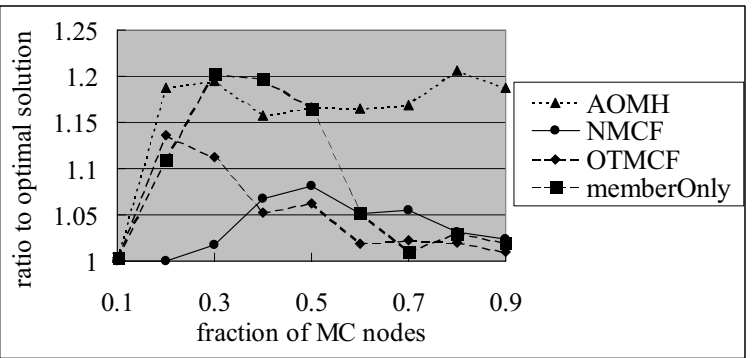

Figure 5. Ratio to optimal for different approaches

Next, the performance of our approach is compared with AOMH and MemberOnly. The comparison is depicted in Fig 6 (b). To further investigate the effectiveness of multicast trees constructed by each approach, especially for observing the light-tree cost of our mechanism compared to the optimal, we design an optimal algorithm using the brute-force mechanism to calculate the minimum-cost of a light-tree. Due to the high computational complexity of the brute-force solution, we use a smaller network in this experiment. 200 multicast sessions with group size 13 are randomly generated in a 15-node network. We then apply OTMCF, NMCF, $\mathrm{AOMH}$, memberOnly, and the brute-force mechanism to construct light-trees and calculate the ratio of the tree cost of each approach to that of the brute-force optimal one. Fig. 5 shows the ratio to the optimal solution for each approach. The tree cost of NMCF is very close to the optimal solution (within $1 \%$ error ratio). AOMH has relatively large error ratio, especially when the percentage of MC nodes exceeds $20 \%$. MemberOnly also has a large error ratio when the fraction of MC nodes is small.

\subsection{Maximum Number of Wavelengths Used per Link}

This experiment is performed to compare the maximum number of wavelengths used per link for a 
session by each approach. Again, we consider MemberOnly, AOMH, OTMCF, and NMCF with KMB as the Steiner tree heuristic, and simulate in a 50-node flat network generated by GT-ITM.

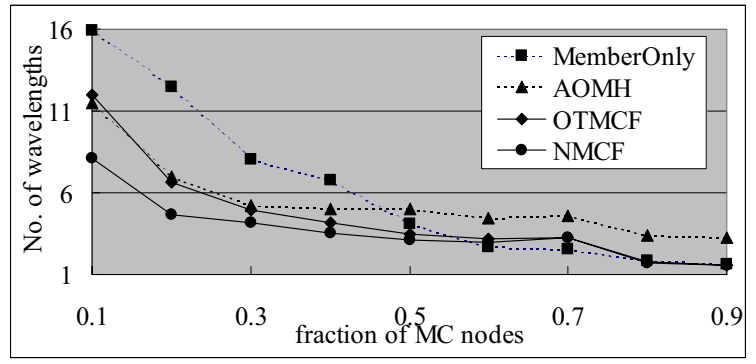

(a) Number of wavelengths

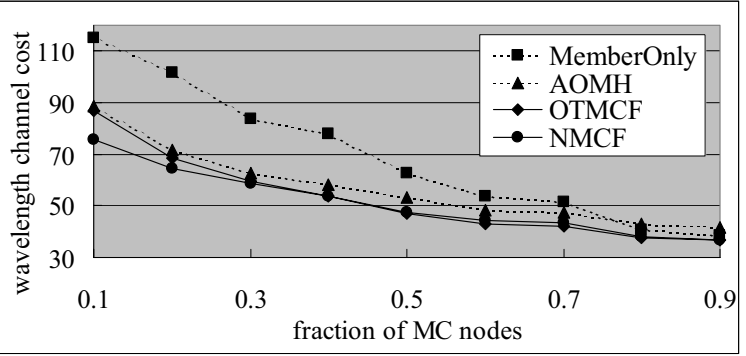

(b) Wavelength channel cost

Figure 6. Performance comparison with AOMH and MemberOnly

Fig. 6 (a) shows the maximum number of wavelengths used per link (or wavelengths required) for the four approaches. Note that we also show the wavelength channel cost of each approach in Fig. 6 (b) for comparison. It can be observed that the number of wavelengths required by a given multicast tree always decreases as the fraction of MC nodes increases for all approaches. When the percentage of $\mathrm{MC}$ nodes in the network exceeds $50 \%$, MemberOnly can construct light-trees efficiently in terms of number of wavelengths required. However its performance degrades rapidly when the percentage of MC nodes in the network becomes small. Of these four approaches, NMCF always achieve the best performance. For example, in most cases, the number of wavelengths used by MemberOnly is more than twice the wavelengths required by $\mathrm{NMCF}$. Considering the wavelength channel cost required, NMCF always uses the least wavelength channels of these four. Thus we can conclude that NMCF is the most efficient way to construct all-optical multicast trees.

\section{Concluding Remarks}

This paper has proposed two mechanisms to construct MC-based light-trees with minimum wavelength channel cost for a multicast session in optical networks with sparse light splitting. Our mechanism eliminates the requirement of rerouting. Based on this, we developed an approximation algorithm for constructing a minimum-cost MC-tree. We have developed two light-tree construction algorithms based on two different on-tree MC nodes selection philosophies. We have also performed simulations to compare our mechanism with AOMH and MemberOnly. The results show that NMCF is the best choice for multicast in sparse splitting optical networks. In all cases, our mechanism requires the least wavelength channel cost and the smallest number of wavelengths per link, as compared with existing work.

\section{Acknowledgement}

This work was supported in part by MOE program for Promoting Academic Excellence of Universities under grant number 89E-FA06-2-4-7, and in part by the National Science Council, Taiwan, under grant number NCS91-2213-E-002-067.

\section{References}

[1] L. H. Sahasrabuddhe and B. Mukherjee, "Light-trees: Optical Multicasting for Improved Performance in Wavelength-Routed Networks," IEEE Communications Magazine, vol. 37, pp. 67-73, Feb. 1999.

[2] X. Zhang, et al., "Constrained Multicast Routing in WDM Network with Sparse Light Splitting," IEEE Journal of Lightwave Technology, vol. 18, no. 12, Dec. 2000

[3] S. Yan, et al., "Route Optimization of Multicast Sessions in Sparse Light-Splitting Optical Networks," IEEE GLOBECOM, 2001

[4] W.- Y. Tseng et al. "All-Optical Multicasting on Wavelength-Routed WDM Networks with Partial Replication,” IEEE ICC '01, Helsinki, Finland, June 2001.

[5] N. Sreenath, "Virtual Source based Multicast Routing in WDM Networks with Sparse-Light Splitting," IEEE High Performance Switching and Routing, 2001

[6] C. Y. Hsieh, "All Optical Multicast in Dense Wavelength Division Multiplexing (DWDM) Networks," Master Thesis, National Taiwan University, June 2002. 